\title{
Which factors are associated with bone marrow oedema suspicious of axial spondyloarthritis as detected by MRI in the sacroiliac joints and the spine in the general population?
}

\author{
Xenofon Baraliakos (1) , ${ }^{1}$ Adrian Richter, ${ }_{1}^{2,3}$ Daniel Feldmann, ${ }^{1}$ Anne Ott, ${ }^{4}$ \\ Robin Buelow, ${ }^{5}$ Carsten 0 Schmidt, ${ }^{6}$ Juergen Braun ${ }^{7}$
}

Handling editor Josef $\mathrm{S}$
Smolen

${ }^{1}$ Rheumazentrum Ruhrgebiet, Ruhr University Bochum, Herne, Nordrhein-Westfalen, Germany Institute of Community Medicine, University of Greifswald, Greifswald, Mecklenburg-Vorpommern, Germany

German Rheumatism Research Center Berlin, Berlin, Germany ${ }^{4}$ Rheumazentrum Ruhrgebiet, Ruhr University Bochum, Herne, Germany

${ }^{5}$ Institute of Diagnostic Radiology and Neuroradiology, University Medicine Greifswald, Greifswald, Germany

${ }^{6}$ Institute for Community Medicine, University of Greifswald, Greifswald, Germany

Rheumazentrum Ruhrgebiet, Ruhr-University Bochum, Herne, Germany

Correspondence to Dr Xenofon Baraliakos, Rheumazentrum Ruhrgebiet, Herne 44649, NordrheinWestfalen, Germany; baraliakos@me.com

$X B$ and $A R$ contributed equally.

$\operatorname{COS}$ and JB are joint senior authors.

Received 21 July 2020

Revised 3 October 2020

Accepted 26 October 2020

Published Online First

25 November 2020

Check for updates

(c) Author(s) (or their employer(s)) 2021. No commercial re-use. See rights and permissions. Published by BMJ.

To cite: Baraliakos $X$,

Richter A, Feldmann D,

et al. Ann Rheum Dis

2021:80:469-474.

\section{ABSTRACT}

Objective Identify factors associated with presence and extension of spinal and sacroiliac joints (SIJ)-MRI lesions suggestive of axial spondyloarthritis (axSpA) in a population-based cohort (Study of Health in Pomerania) aged $<45$ years.

Methods Spinal (sagittal T1/T2) and SIJ (semicoronal STIR sequences) MRIs were evaluated by two trained blinded readers. The presence (yes/no) and extension (Berlin MRI Score) of bone marrow oedema (BME) were captured. Degenerative spinal lesions were excluded and discrepancies resolved by consensus. Cross-sectional associations between clinical factors and presence/ extension of BME were analysed by logistic/negative binomial regression. Record linkage of claims data was applied to identify participants with axSpA.

Results MRIs of 793 volunteers were evaluated. The presence of SIJ-BME (odds ratio) was strongly associated delivery during the last year $(4.47,1.49-13.41)$. For SIJ-BME extension, associations (incidence rate ratios, $95 \% \mathrm{CI}$ ) were found for delivery ((during last year) 4.52 , 1.48-13.84), human leucocyte antigen (HLA)-B27+ $(2.32,1.30-4.14)$, body mass index ( $25-30 \mathrm{vs}<25 \mathrm{~kg} /$ $\mathrm{m}^{2} ; 1.86$ (1.19-2.89)) and back pain ((last 3 months) 1.55, 1.04-2.31), while for spinal BME, associations were found for age per decade $(1.46,1.13-1.90)$ and physically demanding work $(1.46,1.06-2.00)$. Record linkage was available for 694 (87.5\%) participants and 9/694 (1.3\%) had a record of axSpA (ICD M45.09).

Conclusion These population-based data support the hypothesis of mechanic strain contributing to BME in the general population aged $<45$ years and the role of HLA-B27+ as a severity rather than a susceptibility factor for SIJ-BME.

\section{INTRODUCTION}

Axial spondyloarthritis (axSpA) is a chronic rheumatic disease that is characterised by inflammatory back pain and several other musculoskeletal and extramusculoskeletal disease manifestations and comorbidities. ${ }^{1}$ The classification criteria published in 2009 by the Assessment in Spondyloarthritis International Society (ASAS) have set the scene for a differentiation between the classical ankylosing spondylitis (AS), or radiographic SpA, and the nonradiographic form of $\mathrm{SpA}$, based on the presence or absence of definite radiographic changes in the sacroiliac joints (SIJ). ${ }^{2}$ Conventional radiography

\section{Key messages}

What is already known about this subject?

- There is a relatively high frequency of inflammatory and fatty spinal/inflammatory sacroiliac joints (SIJ) and spinal MRI lesions suggestive of has been suspected.

- The reasons for these false-positive signals are still insufficiently investigated.

What does this study add?

- Human leucocyte antigen (HLA)-B27+, delivery during the last year in female adults and presence of back pain in the last 3 months seem to be the most important predictors for the extent of bone marrow oedema (BME) in the SIJ, while age and physically demanding work seem to be the most relevant predictors for the extent of BME in the spine.

- These data also support the role of HLA-B27 as a severity rather than a susceptibility factor for the occurrence of BME in the SIJ.

How might this impact on clinical practice or future developments?

- There is a different association between the occurrence and extension of BME in the SIJ in persons with high body mass index.

- The associations presented here are relevant for the interpretation of spinal MRIs of young people with back pain in blue collar jobs and their possible referral to rheumatologists for exclusion or confirmation of spondyloarthritis.

and MRI as imaging methods and human leucocyte antigen (HLA)-B27 as a laboratory test play an important role for diagnosis and classification of axSpA. ${ }^{3}$ Recently, ASAS has published updates on the recommendations of how to interprete MRIs of the SIJ and the spine in patients with axSpA. ${ }^{45}$ Yet, recent studies have shown that not only axSpA can be related to such changes in the axial skeleton and that differential diagnosis may sometimes be difficult. ${ }^{6}$ Among others, we have recently demonstrated that there is a large proportion of falsepositive MRIs suggestive of inflammatory activity as seen in axSpA, as well as frequent other MRI changes such as fat lesions, in the axial skeleton in 
the normal population. ${ }^{78}$ Furthermore, these changes may also occur in situations with physical demanding activities ${ }^{9}$ or after pregnancy. ${ }^{10}$

The pathogenesis of axSpA is not fully understood to date but it seems to be largely genetically determined. ${ }^{11}$ HLA-B27 is itself responsible for about $20 \%$ of the total genetic risk ${ }^{11}$ and HLAB27 + individuals carry a 10-20-fold increased risk of developing SpA. ${ }^{12}$ In the last two decades, genetic studies have provided major insights into this topic, by identifying susceptibility alleles including $>100$ established loci which contribute roughly to $10 \%$ of the heritability of the disease, over and above the major effect of HLA-B27. ${ }^{11}$ Although the association of AS with HLAB27 was already reported in $1973,{ }^{12}$ it took more than 30 years to establish its role for the classification, ${ }^{2}$ diagnosis ${ }^{13}$ and the referral ${ }^{14}$ of patients with axSpA. The clinical relevance of HLAB27 has recently also been demonstrated in patients with psoriatic arthritis ${ }^{15}$ and chronic inflammatory bowel disease. ${ }^{16}$

Using data from participants of a large population-based study $<45$ years, ${ }^{17} 18$ we conducted a cross-sectional analysis to identify factors associated with MRI changes that are known to occur frequently in patients with axSpA in the SIJ and the spine.

\section{METHODS}

\section{Study sample}

The population-based project 'Study of Health in Pomerania' (SHIP) comprises the two separate cohorts SHIP and SHIPTREND that are sampled in a north-eastern region of Germany and followed up every 4-6 years. SHIP is part of the Community Medicine Research net of the University Medicine of Greifswald, Germany, and conducts an extensive clinical examination programme in each follow-up of the cohorts ${ }^{17}$

Within the second follow-up of SHIP-2 and the baseline examination of SHIP-TREND-0, whole-body MRI was performed. For the present study, MRIs of the 793 volunteers being $<45$ years at the day of the MRI examination and who had complete MRI sets (both spine and SIJ) were included. The selection of volunteers from the SHIP project has been described in more detail elsewhere. ${ }^{17}$

\section{MRI and reading of images}

MRI was performed by the Department of Diagnostic Radiology and Neuroradiology in at one study site using one MRI device (Magnetom Avanto, Siemens Medical Systems, Erlangen, Germany) and under the same standardised protocol. ${ }^{18}$ For the entire spine T1 (TR $6.760 \mathrm{~ms} / \mathrm{TE} 120 \mathrm{~ms}$, flip angle $150^{\circ}$, slice thickness $4 \mathrm{~mm}$, scan time 2:42 min) and T2 (TR $37.600 \mathrm{~ms} /$ TE $1.060 \mathrm{~ms}$, flip angle $180^{\circ}$, slice thickness $4 \mathrm{~mm}$, scan time 2:04 min) MRI sequences were available in a sagittal and for the SIJ respective STIR (TR $48.910 \mathrm{~ms} / \mathrm{TE} 670 \mathrm{~ms}$, flip angle $180^{\circ}$, slice thickness $5 \mathrm{~mm}$ ) sequences in a semicoronal orientation were available. All images were blinded for additional participant information such as age and gender. Two readers, who first completed a training session of reference images including patients with axSpA, evaluated the MRI independently in a paired fashion to assess bone marrow oedema (BME) (defined as hyperintense signal on T2-weighted and hypointense signal on T1-weighted images in the spine or as hyperintense signal on STIR sequences in the SIJs). The ASAS definitions were used for defining lesions as 'positive'. ${ }^{419}$ Spinal lesions with pathologic changes involving the vertebral endplate or being accompanied by abnormalities of the intervertebral disc (obvious dehydration, protrusion or prolapse) were considered as degenerative and were not counted. In case of disagreement for a lesion being present or not present, both readers adjudicated together in order to reach consensus.

In addition to a the binary approach of lesions being present or absent, quantification of the extension of BME lesions was performed based on the definitions of the Berlin SIJ and spine MRI Score. ${ }^{20}$ Briefly, this score captures BME in a scale of 0-3. Both for the SIJ and the spine, a score of 0 means no BME, while a score of 1,2 and 3 mean $0 \%$ to $\leq 33 \%,>33 \%$ to $\leq 66 \%$ and $>66 \%$ of an SIJ quadrant or and $0 \%$ to $\leq 25 \%,>25 \%$ to $\leq 50 \%$ and $>50 \%$ of a discovertebral unit, respectively. The final scores used for analysis were calculated based on consent of both readers in cases of disagreement.

\section{Collection of clinical information}

Clinical information was collected for age (in years), sex, smoking habit (current smoker, previous smoker, no smoker), mean spinal back pain level in the last 3 months prior to the MRI examination (on a numerical rating scale (NRS) rated 0-10), high-sensitivity $\mathrm{C}$ reactive protein (hsCRP, in $\mathrm{mg} / \mathrm{dL}$ ), HLA-B27 status (positive or negative), body mass index (BMI) (categorised according to the definitions of WHO, WHO to normal or underweight, overweight and obese), blue-collar or white-collar job, and whether the (female) patients had given birth within the last 12 months prior to the MRI examination.

\section{Linkage of claims data}

Claims data were available from the regional Association of Statutory Health Insurance including ICD-10 codes between 2002 and 2018. These data were used to obtain all ICD-10 codes of AS M45.xx including the quarterly period after the SHIP examination has been conducted.

As the claims data and those of the SHIP study have no common key for linkage, we applied record linkage as proposed in Vatsalan $e \mathrm{al}^{21}$ based on: surname, name, date of birth and sex of participants with linkage consent. The names were normalised to upper-case letters and by removal of special characters, the indexing of candidate pairs was blocked via birth date. After comparison of candidate pairs, respective claims data were available for 694 of 769 (90.2\%) participants who consented (769 of $793,97.0 \%)$ in record linkage.

\section{Statistical analysis}

Descriptive measures (mean, median) are shown with SD, minimum and maximum. Frequencies and percentages are provided for categorical data $(\mathrm{N})$. For all variables, the amount of missing values is reported.

The outcomes of affected SIJ quadrants or spinal segments are count data and were, therefore, modelled using negative binomial regression. The decision for a negative binomial regression model versus a Poisson model was based on likelihood ratio test in all outcome categories (SIJ, spine) and in the complete data. Effect estimates of the regression models were exponentiated resulting in incidence rate ratios (IRRs). ${ }^{22}$ Associations for the presence of BME was modelled using multiple logistic regression (BME yes/no).

Missing values were accounted for by multiple imputations using the R-package mice. ${ }^{23}$ Given the low number of missing values in each variable, $\mathrm{m}=10$ imputations were considered sufficient. The imputation model comprised all variables of the analysis model. Imputations for categorical variables were conducted using logistic or generalised logistic regression; imputations of numerical variables were conducted using predictive means. All results from the regression models are pooled over all 
imputations according to Rubins' rule. ${ }^{24}$ The variance increase due to missing data was highest for HLA-B27 with 3.3\%. We found no relevant difference between observations with complete data on those with incomplete data (data not shown).

In a sensitivity analysis we added information on known ICD-10 codes (M45.xx) to the regression models to examine changes and the robustness of results.

The interpretation of results is conducted in compliance with recommendations to avoid dichotomisation into statistically significant and insignificant results. ${ }^{25}$ The use of $\mathrm{p}$ values is restricted to model diagnostics; all effects are described using effect estimates and $95 \%$ CIs.

\section{RESULTS}

A total of 793 MRIs of volunteers were evaluated by the two readers, with mean age $37.3 \pm 6.3$ years, $49.4 \%$ male, $67(8.4 \%)$ HLA-B27+, 53 (6.7\%) with hsCRP $>0.5 \mathrm{mg} / \mathrm{dL}, 451$ (56.9\%) with back pain in the last 3 months, (228 (28.8\%) with back pain $\geq 4 / 10$ on an NRS) were available. A physically demanding job was reported by 283 individuals (35.7\%), 511 (64.3\%) reported to work at a desktop. More than half $(\mathrm{n}=436)$ of the participants $(55 \%)$ had a BMI $>25 \mathrm{~kg} / \mathrm{m}^{2}$, and 247 were current smokers (31.1\%). Delivery in the last year before the MRI examination was reported by 16 females (5\%). Average physical activity $>1$ hour/day was reported by 653 subjects (82.4\%). All characteristics including the numbers of missing values are shown in the table 1.

For the extension of BME on SIJ-MRIs, the largest IRR (95\% CI) were found for delivery in the last year: 4.52 (1.48 to 13.84), HLA-B27+ : 2.32 (1.30 to 4.14 ) and BMI $25-30$ versus $<25 \mathrm{~kg} /$ $\mathrm{m}^{2}: 1.86$ (1.19 to 2.89$)$. For the extension of BME on spinal MRIs, IRRs were overall lower in size with the largest effects found for age per decade increase: 1.46 (1.13 to 1.90$)$ and physically demanding work: 1.46 (1.06 to 2.00), see figure 1 .

Regarding the presence of positive lesions on MRI only for the occurrence of delivery during the last year a strong association with SIJ-BME was found in females, with an OR of 4.47 (95\% CI: 1.49 to 13.41 ) (figure 1 ).

Finally, in the comparison between SIJ and spinal MRIs, participants with back pain in the last 3 months $(62.5 \%$ vs 56.9\%) had more often SIJ BME than spinal BME, while spinal BME was more frequent than SIJ BME in participants working at a desktop ( $61.5 \%$ vs $54.4 \%)$, while smokers (66.9\% vs $63.8 \%)$ and participants with back pain in the last 3 months $(62.5 \%$ vs $56.9 \%$ ) had more often SIJ BME than spinal BME, respectively, (data not shown).

In a subset of participants of the SHIP cohorts, information on ICD codes of all participants who consented to record linkage was available from the claims data: in 9/694 (1.3\%) the ICD-10 code M45.09 (AS without specified location) was documented prior to the SHIP examination. However, an affirmation of the 'real' diagnoses of AS or axSpA by an expert was not obtained, since this was not part of the study protocol.

Results from sensitivity analyses did not reveal relevant differences to the results presented above. The largest change was found regarding the IRR for HLA-B27+ in SIJ-BME which decreased from $2.32(1.30 ; 4.14)$ to $2.21(1.24 ; 3.95)$.

\section{DISCUSSION}

In this population-based study, HLA-B27+, delivery during the last year in female participants and presence of back pain in the last 3 months were the most important predictors for the extent of BME in the SIJ, while age and physically demanding work
Table 1 Clinical, demographic and imaging characteristics of the 793 participants

\begin{tabular}{|c|c|}
\hline Clinical, demographic and imaging characteristics & All $(n=793)$ \\
\hline \multicolumn{2}{|l|}{ Age (years) } \\
\hline Mean (SD) & $37.3(6.3)$ \\
\hline Median (min, max) & $39.0(21.0-45.0)$ \\
\hline \multicolumn{2}{|l|}{ Sex } \\
\hline Females & $401(50.6 \%)$ \\
\hline Males & $392(49.4 \%)$ \\
\hline \multicolumn{2}{|l|}{ HLA-B27 } \\
\hline Negative & $689(86.9 \%)$ \\
\hline Positive & $67(8.4 \%)$ \\
\hline Missing & $37(4.7 \%)$ \\
\hline \multicolumn{2}{|l|}{ hsCRP $>0.5 \mathrm{mg} / \mathrm{dL}$} \\
\hline No & $708(89.3 \%)$ \\
\hline Yes & $53(6.7 \%)$ \\
\hline Missing & $32(4.0 \%)$ \\
\hline \multicolumn{2}{|l|}{ Back pain in last 3 month } \\
\hline No & $341(43.0 \%)$ \\
\hline Yes & $451(56.9 \%)$ \\
\hline Missing & $1(0.1 \%)$ \\
\hline \multicolumn{2}{|l|}{ Back pain in last 3 months $\geq 4$ (NRS) } \\
\hline No & $228(28.8 \%)$ \\
\hline Yes & $564(71.1 \%)$ \\
\hline Missing & $1(0.1 \%)$ \\
\hline \multicolumn{2}{|l|}{ Physically demanding job } \\
\hline No & $507(63.9 \%)$ \\
\hline Yes & $283(35.7 \%)$ \\
\hline Missing & $3(0.4 \%)$ \\
\hline \multicolumn{2}{|l|}{ Work at desktop } \\
\hline No & $511(64.4 \%)$ \\
\hline Yes & $270(34.0 \%)$ \\
\hline Missing & $12(1.5 \%)$ \\
\hline \multicolumn{2}{|l|}{ BMI categories } \\
\hline$<25$ & $357(45.0 \%)$ \\
\hline 25 to $<30$ & $287(36.2 \%)$ \\
\hline$\geq 30$ & $149(18.8 \%)$ \\
\hline \multicolumn{2}{|l|}{ Smoking } \\
\hline Never & $295(37.2 \%)$ \\
\hline Previous & $250(31.5 \%)$ \\
\hline Current & $247(31.1 \%)$ \\
\hline Missing & $1(0.1 \%)$ \\
\hline \multicolumn{2}{|l|}{ Birth within 1 year prior to the MRI examination } \\
\hline No & $385(96 \%)$ \\
\hline Yes & $16(4.0 \%)$ \\
\hline \multicolumn{2}{|l|}{ Average physical activity (annual) } \\
\hline$>2$ hours & $167(21.1 \%)$ \\
\hline $1-2$ hours & $486(61.3 \%)$ \\
\hline$<1$ hour & $140(17.7 \%)$ \\
\hline \multicolumn{2}{|l|}{ SIJ: number of affected quadrants } \\
\hline Mean (SD) & $0.236(0.622)$ \\
\hline Median (min, max) & $0(0-8.00)$ \\
\hline \multicolumn{2}{|l|}{ SIJ: Berlin Score } \\
\hline Mean (SD) & $0.288(1.08)$ \\
\hline Median (min, max) & $0(0-24.0)$ \\
\hline \multicolumn{2}{|l|}{ Spine: number of affected segments } \\
\hline Mean (SD) & $0.456(0.911)$ \\
\hline Median (min, max) & $0(0-6.00)$ \\
\hline \multicolumn{2}{|l|}{ Spine: Berlin Score } \\
\hline Mean (SD) & $0.498(1.04)$ \\
\hline Median (min, max) & $0(0-8.00)$ \\
\hline
\end{tabular}




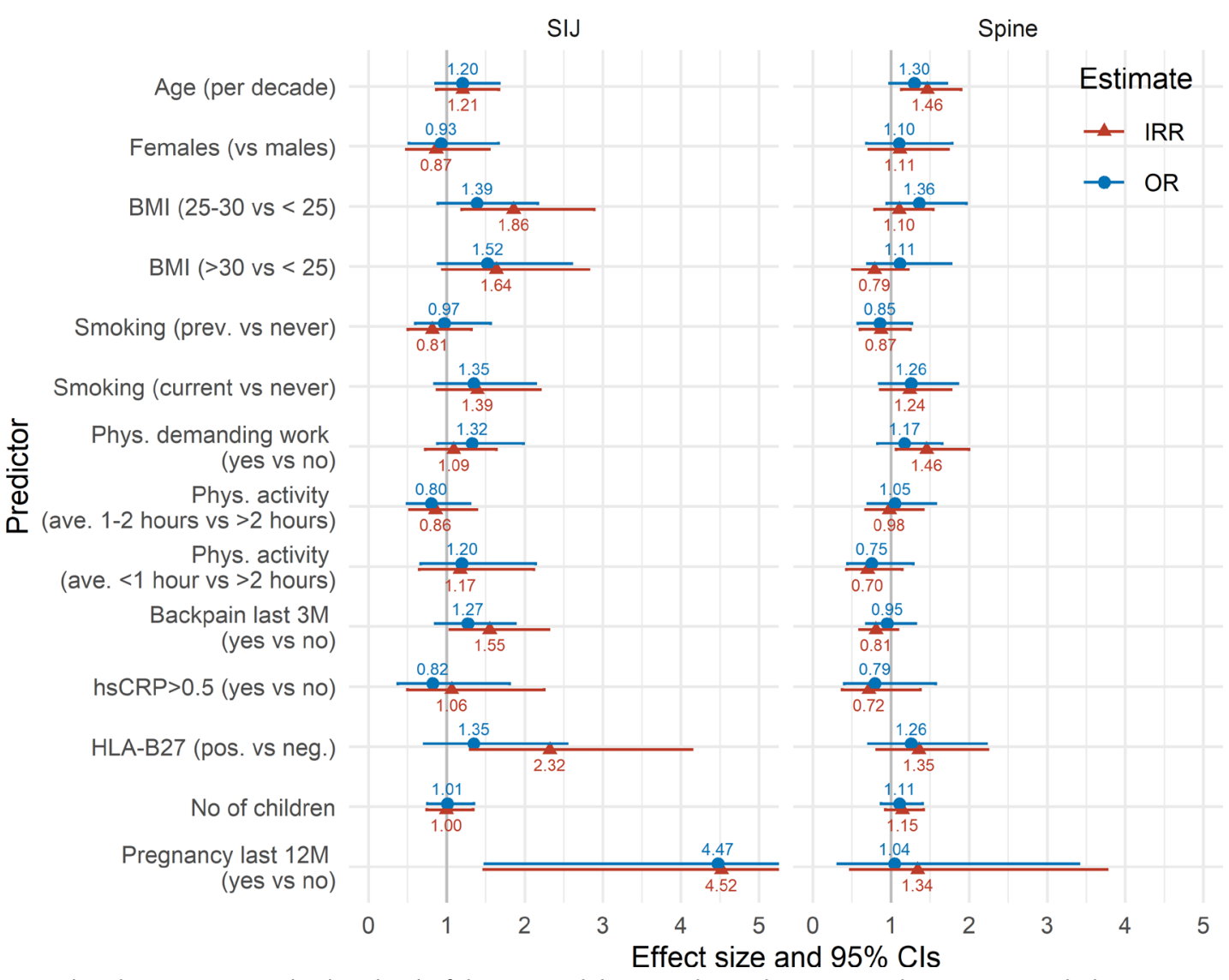

Figure 1 Esimates (incidence rate ratios (IRR) and OR) of the assessed demographic and occupational parameters with the occurrence of bone marrow oedema in the sacroiliac joints (SIJ) and the spine. BMI, bone mass index; hsCRP, high-sensitivity C reactive protein; HLA-B27, human leucocyte antigene B27.

were the most relevant predictors for the extent of BME in the spine.

These data support the hypothesis that mechanic strain contributes to BME found in the general population in subjects not diagnosed with SpA. In addition, HLA-B27+ was associated with the extent of BME but only contributes to a minor extent as a susceptibility factor for BME in the SIJ, while no association with spinal MRI changes was detected. Although the link between mechanically induced inflammation, genes and the immune system still needs to be elucidated, our data suggest, for the first time, that the recently supported ${ }^{26-28}$ hypothesis coming from studies with patients also applies on a general population level.

Our finding of a clear association of BME in the SIJ after delivery within a relatively short period of time prior to the performance of the MRI is based on a small number of events $(n=16)$. However, the result is clinically plausible, looking at the reported prevalence of $\mathrm{MRI}^{29}$ and $\mathrm{CT}^{30}$ findings of osteitis condensans ilii (OCI) in young patients with symptoms of low back pain. In a recent Belgian study, ${ }^{10} 27$ (77\%) out of 35 women developed sacroiliac BME immediately postpartum, with $60 \%$ fulfilling the ASAS definition of a positive MRI in axSpA. Importantly, after 6 months, BME was still present in $46 \%$ of the participants. Whether and how OCI, a condition with a rather obvious mechanic pathogenesis, is associated with axSpA is unclear at present. In a recent study on OCI from Berlin, sacroiliac BME occurred frequently and equally often in both groups but only $7.4 \%$ of patients with OCI developed erosions in the SIJ even though about $35 \%$ of women with OCI were HLA-B27+. ${ }^{31}$
The association of back pain with the extension of BME in the SIJ but not the spine, even though the latter was more frequent, confirms its pathologic relevance related to the development of SpA, including the size of such lesions. ${ }^{4}$ Nevertheless, it needs to be taken into account that the participants in this cohort study were not patients complaining about chronic or inflammatory back pain but were individuals from the general population. Thus, it may be expected that about $6 \%$ of the participants in this study had inflammatory back pain, ${ }^{32}$ and based on a population prevalence of axSpA of $0.5 \%-1 \%$, about four to eight patients may also have had axSpA. ${ }^{33}$ Since it was not planned in this cohort study to directly diagnose patients, we cannot provide data on classification. Nevertheless, results obtained from record linkage of claims data showed that there were no established cases of axSpA in this study population, even though nine participants had been given the ICD code M45.09 somewhere in outpatient care at least once. This result is in line with the clinical experience that axSpA cases will be identified late or not at all. ${ }^{34}$ These nine subjects were more likely to be HLA$\mathrm{B} 27+$ and to have MRI changes in the SIJ (data not shown); this is consistent with this interpretation. It is also worth mentioning that the successful use of non-steroidal anti-rheumatic drugs (NSAIDs), which is not only part of the classification criteria for axSpA but sometimes also used as a diagnostic tool, was only present in a few patients and not associated with the presence of BME in this study (data not shown).

Furthermore, the prevalence of back pain, known to increase with age ${ }^{35}$ during the first life decades, ${ }^{36}$ was confirmed for subjects below the age of 45 in our study. The result that 
participants working at a desktop had more often BME in the spine than in the SIJ may be methodologically explained since the differentiation of the severity of the physical workload (eg, blue or white collar) was not very sharp in this study. However, the finding that the extent of spinal BME was significantly associated with physically demanding work makes sense from the clinical point of view and is consistent with previous work. ${ }^{37}$ Taken together, these results confirm that BME does occur in the younger general population with no established diagnosis of axSpA. Since physically demanding work is known to be associated with chronic back pain, ${ }^{38}$ these associations are relevant for the interpretation of spinal MRIs of young people with back pain in blue collar jobs. Other assessments of physical activity showed smaller associations with the occurrence or extent of BME in our study. However, we did not assess the intensity of physical activity in more detail. In another study, physical inactivity over 14 years was shown to be associated with disc degeneration in the thoracic and the lumbar spine. ${ }^{39}$ Similarly, physically demanding work may also have an effect of patients with axSpA. This is not necessarily related to inflammation. ${ }^{40}$

In addition, while the association of back pain with BMI and fat mass is well established, ${ }^{41}$ our data suggest that there is also a different association between the occurrence and extension of BME in the SIJ in persons with high BMI (OR vs IRR, figure 1). This also seems to point in the direction of mechanical strain as a cause of more severe BME and is likely to stimulate research in this area.

Finally, only no or small associations between the occurrence of BME with any other of the investigated factors, such as smoking, hsCRP or female sex were found in our cohort. All of those factors are special interest in the overall topic of SpA-related MRI findings, since they represent established risk factors for low back pain ${ }^{42}$ and also have been found to have an impact on the radiographic course of axSpA. ${ }^{43}$

In conclusion, in this cross-sectional analysis of MRIs of the SIJ and spine of participants of a population-based study, we identified predictors relevant to the presence of BME suggestive of axSpA. Our findings support the hypothesis of a mechanic origin of BME in the general population aged $<45$ years. On the other hand, HLA-B27 was identified to be a severity rather than a susceptibility factor especially for the presence of BME in the SIJ. Longitudinal analyses may be able to demonstrate causal relationships.

Acknowledgements Study of Health in Pomerania is part of the Community Medicine Research Net of the University Medicine Greifswald, which is supported by the Federal State of Mecklenburg-West Pomerania, Germany, as well as by a joint grant from Siemens Healthineers, Erlangen.

Funding The authors have not declared a specific grant for this research from any funding agency in the public, commercial or not-for-profit sectors.

Competing interests None declared.

Patient and public involvement Patients and/or the public were not involved in the design, or conduct, or reporting, or dissemination plans of this research.

Patient consent for publication Not required.

Ethics approval The study was approved by the local ethics committee of the University Medicine Greifswald.

Provenance and peer review Not commissioned; externally peer reviewed.

Data availability statement Data are available upon reasonable request. All data relevant to the study are included in the article or uploaded as supplementary information. All analyses are described in the manuscript. For additional information, please contact the corresponding author.

ORCID iD

Xenofon Baraliakos http://orcid.org/0000-0002-9475-9362

\section{REFERENCES}

1 Braun J, Sieper J. Ankylosing spondylitis. The Lancet 2007;369:1379-90

2 Rudwaleit M, van der Heijde D, Landewé R, et al. The development of assessment of spondyloarthritis International Society classification criteria for axial spondyloarthritis (Part II): validation and final selection. Ann Rheum Dis 2009;68:777-83.

3 Braun J, Baraliakos X. Imaging of axial spondyloarthritis including ankylosing spondylitis. Ann Rheum Dis 2011;70 Suppl 1:i97-103.

4 Lambert RGW, Bakker PAC, van der Heijde D, et al. Defining active sacroiliitis on MRI for classification of axial spondyloarthritis: update by the ASAS MRI Working group. Ann Rheum Dis 2016;75:1958-63.

5 Maksymowych WP, Lambert RG, Østergaard M, et al. Mri lesions in the sacroiliac joints of patients with spondyloarthritis: an update of definitions and validation by the ASAS MRI Working group. Ann Rheum Dis 2019;78:1550-8.

6 Braun J, Baraliakos X, Buehring B, et al. [Differential diagnosis of axial spondyloarthritis-axSpA mimics]. Z Rheumatol 2019;78:31-42.

7 Baraliakos X, Richter A, Feldmann D, et al. Frequency of MRI changes suggestive of axial spondyloarthritis in the axial skeleton in a large population-based cohort of individuals aged $<45$ years. Ann Rheum Dis 2020;79:186-92.

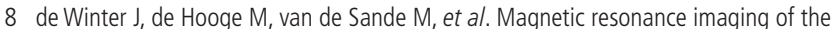
Sacroiliac joints indicating sacroiliitis according to the assessment of spondyloarthritis International Society definition in healthy individuals, runners, and women with postpartum back pain. Arthritis Rheumatol 2018;70:1042-8.

9 Varkas $\mathrm{G}$, de Hooge M, Renson T, et al. Effect of mechanical stress on magnetic resonance imaging of the sacroiliac joints: assessment of military recruits by magnetic resonance imaging study. Rheumatology 2018;57:508-13.

10 Renson T, Depicker A, De Craemer A-S, et al. High prevalence of spondyloarthritis-like MRI lesions in postpartum women: a prospective analysis in relation to maternal, child and birth characteristics. Ann Rheum Dis 2020;79:929-34.

11 Hanson A, Brown MA. Genetics and the causes of ankylosing spondylitis. Rheum Dis Clin North Am 2017:43:401-14.

12 Brewerton DA, Hart FD, Nicholls A, et al. Ankylosing spondylitis and HL-A 27. Lancet 1973:1:904-7

13 van den Berg R, de Hooge M, Rudwaleit M, et al. ASAS modification of the Berlin algorithm for diagnosing axial spondyloarthritis: results from the SPondyloArthritis Caught Early (SPACE)-cohort and from the Assessment of SpondyloArthritis international Society (ASAS)-cohort. Ann Rheum Dis 2013;72:1646-53.

14 Braun A, Gnann H, Saracbasi E, et al. Optimizing the identification of patients with axial spondyloarthritis in primary care--the case for a two-step strategy combining the most relevant clinical items with HLA B27. Rheumatology 2013;52:1418-24.

15 Coates LC, Baraliakos X, Blanco FJ, et al. The phenotype of axial spondyloarthritis: is it dependent on HLA-B27 status? Arthritis Care Res 2020. doi:10.1002/acr.24174. [Epub ahead of print: $26 \mathrm{Feb} 2020]$.

16 Ossum AM, Palm Øyvind, Lunder AK, et al. Ankylosing spondylitis and axial spondyloarthritis in patients with long-term inflammatory bowel disease: results from 20 years of follow-up in the IBSEN study. J Crohns Colitis 2018;12:96-104.

17 Völzke H, Alte D, Schmidt CO, et al. Cohort profile: the study of health in Pomerania. Int J Epidemiol 2011;40:294-307.

18 Hegenscheid K, Kühn JP, Völzke H, et al. Whole-Body magnetic resonance imaging of healthy volunteers: pilot study results from the population-based SHIP study. Rofo 2009;181:748-59.

19 Hermann K-GA, Baraliakos X, van der Heijde DMFM, et al. Descriptions of spinal MRI lesions and definition of a positive MRI of the spine in axial spondyloarthritis: a consensual approach by the ASAS/OMERACT MRI study Group. Ann Rheum Dis 2012;71:1278-88.

20 Baraliakos X, Braun J. Imaging scoring methods in axial spondyloarthritis. Rheum Dis Clin North Am 2016;42:663-78.

21 Vatsalan D, Christen P, Verykios VS. A taxonomy of privacy-preserving record linkage techniques. Inf Syst 2013;38:946-69.

22 Long JS, Freese J. Regression models for categorical dependent variables using Stata: Stata press, 2006.

23 Buuren Svan, Groothuis-Oudshoorn K. mice : Multivariate Imputation by Chained Equations in R. J Stat Softw 2011:45:1-68.

24 Rubin DB. Multiple imputation for survey nonresponse. New York: Wiley, 1987.

25 Wasserstein RL, Schirm AL, Lazar NA. Moving to a world beyond " $p<0.05$ ". Taylor \& Francis, 2019.

26 Braun J, Baraliakos X. Active and chronic sacroilitis, spondylitis and enthesitis, how specific are imaging findings for axial spondyloarthritis? Rheumatology 2019;58:1321-4.

27 Jacques $P$, Lambrecht $S$, Verheugen E, et al. Proof of concept: enthesitis and new bone formation in spondyloarthritis are driven by mechanical strain and stromal cells. Ann Rheum Dis 2014;73:437-45.

28 Cambré I, Gaublomme D, Burssens A, et al. Mechanical strain determines the sitespecific localization of inflammation and tissue damage in arthritis. Nat Commun 2018:9:4613.

29 Eshed I, Lidar M. Mri findings of the Sacroiliac joints in patients with low back pain: alternative diagnosis to inflammatory sacroiliitis. Isr Med Assoc J 2017;19:666-9. 
30 Klang E, Lidar M, Lidar Z, et al. Prevalence and awareness of sacroiliac joint alterations on lumbar spine CT in low back pain patients younger than 40 years. Acta Radiol 2017;58:449-55.

31 Poddubnyy D, Diekhof T, Gobejishvili N, et al. Magnetic resonance imaging of Sacroiliac joints in patients with osteitis Condensans Ilii reveals a typical pattern of lesions relevant for differential diagnosis with axial spondyloarthritis. Arthritis Rheumatol 2018;70.

32 Weisman MH, Witter JP, Reveille JD. The prevalence of inflammatory back pain population-based estimates from the US National health and nutrition examination survey, 2009-10. Ann Rheum Dis 2013;72:369-73.

33 Stolwijk C, Boonen A, van Tubergen A, et al. Epidemiology of spondyloarthritis. Rheum Dis Clin North Am 2012;38:441-76.

34 Redeker I, Callhoff J, Hoffmann F, et al. Determinants of diagnostic delay in axial spondyloarthritis: an analysis based on linked claims and patient-reported survey data. Rheumatology 2019;58:1634-8.

35 Hoy D, March L, Brooks P, et al. The global burden of low back pain: estimates from the global burden of disease 2010 study. Ann Rheum Dis 2014;73:968-74.

36 Chung CB, Vande Berg BC, Tavernier T, et al. End plate marrow changes in the asymptomatic lumbosacral spine: frequency, distribution and correlation with age and degenerative changes. Skeletal Radiol 2004;33:399-404.
37 Poddubnyy D, Weineck H, Diekhoff T, et al. Clinical and imaging characteristics of osteitis condensans ilii as compared with axial spondyloarthritis. Rheumatology 2020. doi:10.1093/rheumatology/keaa175. [Epub ahead of print: 23 May 2020].

38 Mikkonen P, Viikari-Juntura E, Remes J, et al. Physical workload and risk of low back pain in adolescence. Occup Environ Med 2012;69:284-90.

39 Maurer $\mathrm{E}$, Klinger $\mathrm{C}$, Lorbeer $\mathrm{R}$, et al. Long-Term effect of physical inactivity on thoracic and lumbar disc degeneration-an MRI-based analysis of 385 individuals from the general population. Spine J 2020;20:1386-1396.

40 Ramiro S, Landewé $R$, van Tubergen A, et al. Lifestyle factors may modify the effect of disease activity on radiographic progression in patients with ankylosing spondylitis: a longitudinal analysis. RMD Open 2015;1:e000153.

41 Hussain SM, Urquhart DM, Wang Y, et al. Fat mass and fat distribution are associated with low back pain intensity and disability: results from a cohort study. Arthritis Res Ther 2017;19:26.

42 Hartvigsen J, Hancock MJ, Kongsted A, et al. What low back pain is and why we need to pay attention. Lancet 2018;391:2356-67.

43 Poddubnyy D, Haibel $\mathrm{H}$, Listing J, et al. Baseline radiographic damage, elevated acute-phase reactant levels, and cigarette smoking status predict spinal radiographic progression in early axial spondylarthritis. Arthritis Rheum 2012;64:1388-98. 